Meta

Journal des traducteurs

Translators' Journal

\title{
Traduction du Psautier
}

La strophique des psaumes

\section{Jeannine Bélanger}

Volume 15, numéro 1, mars 1970

La traduction religieuse

URI : https://id.erudit.org/iderudit/002034ar

DOI : https://doi.org/10.7202/002034ar

Aller au sommaire du numéro

Éditeur(s)

Les Presses de l'Université de Montréal

\section{ISSN}

0026-0452 (imprimé)

1492-1421 (numérique)

Découvrir la revue

\section{Citer cet article}

Bélanger, J. (1970). Traduction du Psautier : la strophique des psaumes. Meta, 15(1), 18-25. https://doi.org/10.7202/002034ar d'utilisation que vous pouvez consulter en ligne.

https://apropos.erudit.org/fr/usagers/politique-dutilisation/ 


\section{TRADUCTION DU PSAUTIER \\ La strophique des jsaumes}

Notre immense projet, $\mathrm{D}^{\mathrm{r}}$ Watson Kirkconnell et moi-même, d'un Psautier diglotte en vers, c'est-à-dire d'une traduction juxtalinéaire, dans les langues française et anglaise, des cent cinquante poèmes bibliques, vétérotestamentaires qui ont nom, en hébreu, $t^{e}$ hillìm (Louanges), en français et en anglais Psaumes et Psalms, soulevait et pose forcément à nouveau, avec acuité, tout le problème de la strophe ou stance sémitique ancienne.

On le sait, mon collègue l'a rappelé ${ }^{1}$, la métrique des Psaumes compte des versets de trois plus trois, trois plus quatre, quatre plus trois, quatre plus quatre, voire de trois plus deux et quatre plus deux accents ou arsis, qui se succèdent d'autant moins savamment que l'on remonte, pour la composition, vers l'âge hérö̈que. Dans les chants particulièrement archaïques, mettons au début du premier millénaire avant Jésus-Christ, donc qui ont subi davantage l'usure des siècles, l'agencement prosodique est simple; on ne viendra que tard, vers le $\mathrm{V}^{e}$ ou le $\mathrm{IV}^{\mathrm{e}}$ siècle avant notre ère, à ces factures-acrostiches où tout le morceau s'ordonne sur des espèces de «laisses » ou suites de versets dont chacune commence par une même lettre de l'alphabet, tel le long Psaume 119 dit de la Loi.

Mais, une fois circonscrite l'allure du verset, scandés les battements qui marquent, ligne à ligne, la respiration interne du poème, faut-il aller au-delà et reconnaître, derrière les mètres serrés, de véritables groupes plus vastes de ces stiques ou stiches, tantôt épousant la même cadence, tantôt passant d'une mesure à l'autre, perceptibles seulement à la rupure du sens ou à l'achèvement musical de la période?

Entre autres techniciens, Podechard ${ }^{2}$, sans rien écrire en général sur la strophe psalmique, «bloque » typographiquement par séries de versets sa traduc-

1. Voir p. 13 et 14.

2. Emmanuel Podechard, p.s.s., le Psautier, Notes critiques, I, Psaumes 1-75, Lyon, Facultés catholiques, 1949, notamment p. 78-79. Voir aussi ibid., Traduction littérale et explication historique, I, Psaumes 1-75, Lyon, Facultés catholiques 1949, p. 79-90. 
tion, en prose, des originaux hébraïques. Déjà, Calès ${ }^{3}$ marquait d'ailleurs, au bout de tel nombre de stiques dont la signification est complète, un arrêt qui en fait vraiment à ses yeux une unité à part. Dès lors, le rythme s'isostrophise ou encore devient plus large ou plus dru, pour retrouver parfois ou laisser perdre tout simplement la cadence initiale. Les auteurs de la Bible de Jérusalem se sont montrés sensibles à ces nuances, et, bien qu'ils transposent en prose, prose, il est vrai, éminemment poétique parce que chaude et colorée, ils ne manquent pas de faire sentir le passage d'une unité strophique à l'autre, et non seulement d'un stiche à l'autre, au long des poèmes psalmiques.

Le secret d'aussi complexes ordonnances prosodiques que d'aucuns croient discerner dans les Psaumes a même paru à ces esprits critiques le fruit d'une prestigieuse arithmétique de la part des bardes sacrés. Dans leurs compositions de destination cultuelle, à la manière des chœurs grecs, où des solistes alternent avec le Peuple, les liturgistes de l'antique Jérusalem auraient procédé par de pieux calculs à chaque opus, dont la numérotation, en tête du poème, correspondrait censément au nombre de versets du Psaume, telles deux ou trois lignes déterminées par exemple reparaissant à intervalles plus ou moins réguliers dans l'alternance de la prière au Temple, de manière à constituer un refrain et à ponctuer souvent un récitatif qui s'en trouve entrecoupé comme d'un hoquet lyrique ${ }^{4}$. Sans doute, l'ensemble dut faire grand effet, quand la voix pure du coryphée s'élevait, croisée par la rumeur de foules orantes, ou tout au moins par les répons d'un groupe imposant de chantres autorisés - cette fonction incombait d'office aux lévites, comme tout le service de l'autel! Mais on ne peut que conjecturer là-dessus, le mystère continuant d'entourer les anciennes cérémonies liturgiques israélites ${ }^{5}$.

Que l'on se penche seulement sur l'auguste et cryptique Psaume $18: \ll \mathrm{Je}$ T'aime tendrement, ma force, ô Seigneur !» Dès le huitième verset, changement de registre. Wattig'ash wattir'ash hâ'âreg. Une allitération à peu près intranslittérable introduit aussitôt une théophanie où le Dieu de l'orage Lui-même Se révèle dans une vision apocalyptique : «La terre, frémissant, chancela». Nouveau changement de ton au verset 17 , et retour à l'ode hymnique ainsi qu'à l'action de grâces : «Il étendit la main, m'assuma hors de l'onde ».

Toute la question d'une traduction en vers ou en prose est là. S'agit-il, oui ou non, de poèmes dans l'hébreu ? C'en sont. Qu'eût dit David ou comment se fussent exprimés, à notre époque et pour nos audiences, les chantres inspirés de l'antique Sion? Et quelle poésie française atteindra jamais à la hauteur de ces originaux? «To import into the garden of English poësy - en l'occurrence :

3. Jean Calès, s.j., le Livre des Psaumes, traduit et commenté, I, Introduction, Psaumes I-LXXII, Paris, Beauchesne, 1936, p. 36-37 et notamment p. 215-234.

4. C'est toute la thèse telle que je la trouve élaborée dans la précieuse collection de carnets manuscrits du regretté James R. Allen, ci-devant pasteur de l'église Saint John the Baptist, de Pointe-Claire, qui a travaillé dix-sept ans sur la strophique des Psaumes et dont je compte bien faire mieux connaître, à titre posthume, hélas! l'œuvre critique non moins savante qu'originale.

5. E. Werner, "Music", The Interpreter's Dictionary of the Bible, New York, Abingdon F'ress, 1962, III, p. 457-469. 
into French - exotics that bloom like native flowers ${ }^{6}$, dirait l'illustre traducteur d'Omar, Edward FitzGerald : voilà le hic. Un génie qui ose, et puisse s'équiparer aux créateurs originaux de la psalmographie biblique, on ne nous boudera pas, j'espère, de l'appeler du moins ici de tous nos vœux ?

Et ce, encore qu'au diapason de nos temps, François Chalet, dans Cris d'hommes ${ }^{7}$, prétend adapter les formules psalmiques séculaires à l'oraison contemporaine, non seulement de l'élite mais de la masse. Voici, à la moderne, sa version du Psaume 38, verset 18 : «Toute la journée, j'en ai marre!» Claudel ${ }^{8}$, humiliant la grammaire avec un solécisme toutefois bien glorieux d'une pareille signature, s'écrie dans sa transposition du Psaume 6, verset 3: «Seigneur, ... c'est Votre guérison que j'ai besoin! » On songe à la grande Edna St. Vincent Millay, dans la relativement récente traduction juxtalinéaire où elle anglicise ou américanise - une fois pour toutes, non moins librement, Baudelaire, avec le brio qu'on lui connaît :

Les riches plafonds,

Les miroirs profonds,

La splendeur orientale...

Ceilings richly wrought,

Mirrors deep as thought,

Chacun de nous porte son propre Psautier, bien personnel, au ventre, proclamait naguère André Chouraqui. Certes, tout homme a droit au marbre ou à la verrière, si c'est pour en immortaliser les sanglots qu'il a dans le cour. Et puis après ? Qu'importe que le peintre se damne, si le vitrail qu'il cuit est un beau vitrail ! (Jean Cocteau).

Je m'attarde avec prédilection sur le cher Psaume 18. Il semble que le stique, géminé d'ailleurs en distiques, s'y présente très régulièrement, trois plus trois accents à la ligne. Sur quoi fonder alors une division strophique ? On le découpe d'ordinaire, quand on l'imprime, en quatorze simili-huitains, selon l'original sémitique, même si des théoriciens s'obstinent à sectionner le Psaume différemment, ou refusent toujours d'y reconnaître aucune catégorie de strophes. Trois parties dans ce chant, protestent pourtant les fervents d'une analyse moins formelle, et plus en profondeur, du vétuste cantique. L'ode du début, cri d'exaltation et de victoire; véritable ovation toute personnelle, où le «hourra » millénaire du Peuple de la Bible retentit comme un écho. Puis la théophanie. Enfin, poursuite de la $b^{e}$ râkâh ou bénédiction inaugurale, à la manière d'une louange délirante.

Partout la stance, en notre langue, a intérêt à épouser dans une traduction rythmique les grandes mesures classiques du lyrisme français, - l'anglais s'y conforme du reste dans la traduction Kirkconnell, cette langue permettant maintes dérogations traditionnellement propres au génie de la race : double rime ou distique final ${ }^{10}$, et ainsi de suite. Voici les principales formules strophiques utilisées

6. Préface des voluptueux et vénérables Rubaiyat ou quatrains persans, dont l'incomparable traducteur a donné quatre transpositions successives en langue anglaise, la première ayant fait sa gloire.

7. Francois Chalet, Cris d'hommes, Les Psaumes, Essai d'adaptation pour notre temps, Paris, Les Editions ouvrières, 1966, p. 41.

8. Paul Claudel, Psaumes, Traductions 1918-1959, Paris, Desclée de Brouwer, 1966, p. 31.

9. "L'invitation au voyage/Invitation to the Voyage", dans Gordon Dillon et Edna St. Vincent Millay, les Fleurs du mal/Flowers of Evil (Charles Baudelaire), New York, Washington Square Press, 1962, p. 64-65.

10. En l'occurrence à l'hémistrophe : versets 9-10. 
plus loin - on me pardonnera ce très bref excursus dans le jardin clos des initiés :

\begin{tabular}{|c|c|c|}
\hline I. Versets & $\begin{array}{l}3-4 \\
5-7\end{array}$ & $\begin{array}{l}\text { aabccb } \\
\text { ababccb }\end{array}$ \\
\hline II. & $\begin{array}{r}8-12 \\
13-16\end{array}$ & $\begin{array}{l}\text { ababccdeed } \\
\text { ababccdeed }\end{array}$ \\
\hline III. & $\begin{array}{l}17-20 \\
21-24 \\
25-28 \\
29-31 \\
32-34 \\
35-37 \\
38-40 \\
41-43 \\
44-46 \\
47-49 \\
50-51\end{array}$ & $\begin{array}{l}\text { ababccb } \\
\text { ababccb } \\
\text { ababccb } \\
\text { ababccb } \\
\text { abaab } \\
\text { ababccb } \\
\text { aabccb } \\
\text { aabccb } \\
\text { ababccb } \\
\text { aabccb } \\
\text { abaab }\end{array}$ \\
\hline
\end{tabular}

Mais un tel squelette ne va prendre réellement chair et vie que dans le contexte des deux versions, française et anglaise, juxtaposées en un parallélisme délibérément rigoureux, voire percutant :

Je T'aime tendrement, ma force, ô Seigneur ! Pierre

3 granitique, mon Dieu, ma citadelle altière,

le roc où j'ai rempart, et mon libérateur,

c'est le Seigneur; oui, la corne de mon prestige,

ma tour, mon bouclier, digne, ce Seigneur, dis-je,

4 de toute gloire, en qui j'échappe à l'agresseur !

5 Les vagues de la mer montaient : d'une homicide troupe le flot croissant m'assaillait coup sur coup.

${ }^{6}$ Les chaînes du shéol serraient leur noud perfide, les pièges de la mort bâillant sous moi leur trou.

7 Je priai le Seigneur, du fond de ma détresse, que mon Dieu, de Son temple, à ma clameur S'abaisse, et mon cri devers Lui L'aura trouvé debout.

${ }^{8}$ La terre, frémissant, chancela; les assises

mêmes des monts, alors, on les vit s'émouvoir.

De Lui seul, la colère exerce ces emprises :

${ }^{9} \mathrm{Sa}$ narine et $\mathrm{Sa}$ bouche exhalaient un feu noir.
I love Thee, Lord,

Thou pillar of my might.

$3 \mathrm{He}$ is my rock, my fortress, my delight.

The horn of my salvation and my shield.

My cry for succour to the Lord is raised

For he is ever worthy to be praised

4 And I become a victor in the field.

5 The waves of death encompassed me about,

The torrents of perdition billowed near.

6 The cords of Sheol bound my soul devout,

The snares of death enveloped me in fear.

7 In my distress, I called upon the Lord

Nor in His temple was my voice ignored,

My cry ascending reached His holy ear.

8 Then the earth trembled in dismay benumbing.

As deep foundations of the mountains shake;

They quaked before the anger of His coming.

9 Smoke from His nostrils glided like a snake, 
des charbons embrasés dont la chaleur dévore.

10 Foulant aux pieds la nue où Sa clarté s'instaure,

11 Il venait, chevauchait un sombre chérubin.

Sur les ailes des airs apparut Sa stature :

12 Son pavillon, drapé comme d'une tenture

par l'aqueuse ténèbre, emplissait le lointain.

13 Sous l'éclat de Sa face, une grêle nocturne

de brandons alluma l'ombre, flambeaux épars.

14 Et le Seigneur tonnait dans le ciel taciturne, cette voix du Très-Haut roulant de parts en parts.

15 Sa flèche décochée à travers le silence, combien de fois, Seigneur, comme un éclair s'élance, dispersa l'ennemi, tandis que, par torrent, 16 plus d'un raz de marée étalait les racines du monde, au souffle courroucé de Tes narines,

en meurtrier péril de Ta terreur fumant.

17 Il étendit la main, m'assuma hors de l'onde,

18 m'a sauvé des assauts d'adversaires têtus

en combat inégal, où leur haine profonde

trop aisément, sur moi, aurait eu le dessus.

19 On m'affrontait au jour de ma pire infortune :

20 le Seigneur, octroyant Sa clémence opportune,

pour celui qu'Il aimait déploya Ses saluts.
Consuming fire from His mouth did break,

${ }^{10} \mathrm{He}$ bent the cope of heaven, and descended.

$11 \mathrm{He}$ rode upon a cherub, fierce and warm,

And posted on the pinions of a storm;

12 Great clouds of watery darkness round His form

The tumult of His fiery pomp attended.

13 Out of the brightness from before His face,

Hailstones broke through the clouds, and coals of fire.

$14 \mathrm{His}$ voice of thunder rumbled on apace

As the Most High declared His utter ire.

$15 \mathrm{He}$ sent His arrows forth to drive them out,

He flashed His lightnings and achieved their rout.

The channels of the sea, were bare before Thee,

16 The earth's foundations were with terror floored

At Thy rebuke, $O$ everlasting Lord,

Thy nostrils' blast made day and night adore Thee.

17 He reached down from on high, He drew me forth

18 From many waters of unequal fight

With foes who compassed me from south and north

In hatred that outmatched my feeble might.

19 They came upon me in my day of doom,

20 But God hath set me in an ample room;

He saved my soul, for I am His delight. 
21 Le: Seigneur m’a jugé suivant ma droite cause,

selon. la pureté de mes mains à Ses yeux :

22 car j'observai Sa loi, ce que mon Dieu m'impose,

sans me montrer jamais d'autre chose envieux.

${ }^{23}$ Au contraire, Son code enchantait ma mémoire,

dans Sa parole j'ai placé toute ma gloire,

24 tel de moi me gardant que d'un être odieux.

25 Aussi bien le Seigneur m'a fait miséricorde

pour mes mains sans souillure et ma fuite du mal.

${ }^{26}$ Envers qui prend pitié Ta clémence déborde,

27 avec l'homme innocent Tu Te montres loyal, mais double pour l'auteur d'une entreprise inique.

28 Ton bras vers les petits fut toujours salvifique

et rabaisse l'orgueil de leur hautain rival.

29 N'est-ce point Toi, Seigneur, le rayon de ma lampe?

mon Dieu, l'aube perçant mes noirceurs alentour ?

30 Par Toi je cloue au sol cette troupe qui rampe,

j'enjambe, avec mon Dieu, ce mur sur mon parcour.

31 Le Dieu des forts, Sa voie est sans ombre à mon âme :

les décrets du Seigneur ont passé par la flamme,

Son armure, quiconque allait périr y court.

32 Quel dieu l'est comme Lui, quel Roc offre un asile,

${ }^{33}$ hors le Seigneur, dont la vaillance me ceindra?

Sa grâce raffermit tous mes chemins d'argile,

34 égale aux pieds des cerfs mon pas soudain agile,

et fait que je gravis ces altitudes-là !
21 The Lord rewarded me for righteousness,

According to the cleanness of my hands.

22 For I His mighty laws do still profess

And bind my conduct by His holy bands :

${ }^{23} \mathrm{His}$ judgements and His statutes from of old

$M y$ heart and hands have hastened to uphold,

24 And blameless in His sight my spirit stands.

25 The Lord rewarded me : my deeds He knew

Clean-handed and in righteous duties sure.

26 Thou with the true man art Thyself most true,

27 And with the pure Thou showest Thyself pure.

Yet for the crafty Thou art hard to seek,

28 And Thou dost save a nation that is meek;

The haughty eye Thou blindest beyond cure.

29 With Thee as lamp, O Lord, my way I scan;

Thou hast made clear the darkness to my eye;

30 With Thee to help, I storm the barbican,

And by Thy aid I leap the rampart high.

31 This God, His ways the truest laws employ;

His promises are gold, without alloy;

$\mathrm{He}$ is the shield of all who on Him cry.

32 Who but the Lord is God, who else our rock?

33 This God who girds my body with its might,

Who does not leave my way a laughingstock,

Who makes my feet like hinds' amid the flock,

And holds me safe from falling on the height, 
35 Tu formes pour l'assaut mes deux mains invincibles, entraînes mes deux bras à bander l'arc de fer;

36 Ton souci, loin le moi, déplace toutes cibles,

bouclier sans échec dont Ta droite se sert.

Ta bienveillance accroît ceux qui vont dans Ta charge,

37 ma route, en grand péril, Tu la ramène au large,

mes chevilles, les dresse et me mets à couvert.

38 Je traque l'agresseur, l'ébranle et le décime;

pour lui point de merci, personne qui rédime!

39 Car je vais l'achever à ne plus ressurgir.

Sous mes pieds le voilà comme un objet qu'on foule :

$40 \mathrm{Tu}$ ceins mes reins de force, et me soumets en foule

un ennemi donné pour me l'assujétir.

41 L'adversaire de dos m'est livré, Ta conduite

disperse ce vaincu, je l'accule à la fuite.

42 Il peut gémir : en vain, nul ne fut l'assister.

Son cri vers le Seigneur demeure sans réponse :

43 comme la poudre au sol mon avance l'enfonce,

je le broie à l'instar des glèbes du sentier.

$44 \mathrm{Tu}$ daignes me ravir aux tribus en vacarmes

et subjugue à ma loi chacune nation;

45 un peuple, hier inconnu, rend aujourd'hui les armes,

par Ton pouvoir contraint fait sa tradition.

46 Les fils de l'étranger se disent mes esclaves,

présentent leurs tributs en chaussant mes entraves,

et penauds, remués, vident leur bastion.
35 Who trains my hands for mighty deeds in war,

Who nerves my arms to bend a brazen bow.

36 Thou givest me Thy shield as conqueror,

Thy right hand has supported me below.

Thy saving grace has made my strength complete,

37 A wide room Thou hast spread before my feet,

My steps have never faltered as I go.

38 I follow, and I catch, my enemies,

I strike them down until they cannot rise,

39 I turn not back till they have died in fear.

They fall in death, beneath my feet they lie,

40 For me with battle-strength Thou didst supply,

And caused my foes to sink beneath my spear.

41 Thou mad'st my enemies to turn their backs;

They hated me, but fell at my attacks;

42 They cried for help, but vainly did entreat,

Vain were petitions from these souls that sinned

43 I beat them fine as dust before the wind,

I trod them down like mud upon the street.

44 Thou hast delivered me from warfare's slough

And placed me in the front of every nation;

45 A people that I knew not serves me now,

And foreign nations seek my approbation;

46 They hear my voice and they at once obey;

And foreigners, all faint of heart today,

Come trembling from their forts in agitation. 
47 Béni soit le Seigneur, Pierre de ma revanche, dont la toute-puissance à ma rescousse penche;

48 c'est le Dieu fort, mon triomphe sur l'ennemi.

49 Les peuples, Il les rend de mon joug tributaires :

Tu miélèves au combat, ravales contre terres

l'homme de violence en tutelle réduit.

$50 \mathrm{Je}$ voudrais T'applaudir, Seigneur, devant la face

de tout peuple et jouer un psaume en Ton honneur :

51 e Fameux sont les exploits de David par Sa grâce.

* Il fait miséricorde à ce prince, et la race

« de Son oint pour jamais goûtera $\mathrm{Sa}$ faveur. >
47 Now lives the Lord.

All-blessèd be my rock.

Thou hast brought down my foes that flout and mock.

48 Exalted be the God of my salvation,

49 Who rescues me from enemies that rage,

Who lifts me high above a warlike age,

Delivering me from men of depredation.

50 Among the Gentiles, Lord, I praise Thy name,

Sing praises to the God that $I$ adore.

51 Great triumph to His King He has appointed,

And shown His steadfast love for His anointed,

For David and his heirs for evermore.

Le jeune peintre juif David Silverberg, de l'Université Mount Allison, au Nouveau-Brunswick, a aimablement consenti à illustrer, en primeur, le futur Psautier diglotte de huit eaux-fortes, hautes en couleur, sur la vie du roi David, type par excellence des anciens chantres inspirés d'Israël.

JEANNINE BÉLANGER 